Lehrbuch der Mathematik für Wirtschaftswissenschaften 


\section{Lehrbuch der Mathematik für \\ Wirtschaftswissenschaften}

\section{Auflage}

\section{Herausgegeben von}

Heinz Körth - Carl Otto - Walter Runge - Manfred Schoch

Westdeutscher Verlag 


\section{Originaltitel}

Mathematik für ökonomische und ingenieurökonomische Fachrichtungen, Teil I: Mathematische Grundlagen

\section{Autoren}

Prof. Dr. rer. oec. habil. Horst Baumann, Handelshochschule Leipzig

Prof. Dr. rer. nat. habil. Gustav Burosch, Universität Rostock

Prof. Dr. rer. nat. habil. Werner Dück, Hochschule für Ökonomie „Bruno Leuschner“ Berlin

Dr. rer. nat. Rolf Eilhauer, Pädagogische Hochschule Potsdam

Prof. Dr. rer. nat. habil. Karl-Heinz Elster, Technische Hochschule Ilmenau

Dr. rer. nat. Manfred Freier, Bergakademie Freiberg

Dr. rer. nat. Karl-Heinz Gärtner, Bergakademie Freiberg

Prof. Dr. rer. oec. habil. Heinz Körth, Humboldt-Universität Berlin

Dr. rer. nat. Rainer Lang, Technische Hochschule Ilmenau

Prof. Dr. rer. oec. habil. Carl Otto, Humboldt-Universität Berlin

Heiko Patzer, Technische Hochschule Ilmenau

Prof. Dr. rer. nat. et sc. oec. Walter Runge, Universität Rostock

Prof. Dr. rer. nat. habil. Horst Sachs, Technische Hochschule Ilmenau

Prof. Dr. rer. nat. habil. Manfred Schoch, Bergakademie Freiberg

Dr. rer. oec. Hans-Gerhard Strohe, Humboldt-Universität Berlin

Dr. rer. nat. Manfred Weiß, Hochschule für Verkehrswesen „Friedrich List“ Dresden

\section{Lektor}

Diplomwirtschaftler Wolfgang Adler

\section{Auflage}

ISBN 978-3-531-11210-7 DOI 10.1007/978-3-322-87545-7

Westdeutscher Verlag GmbH, Opladen 1975

Softcover reprint of the hardcover 3rd edition 1975

Copyright by Verlag Die Wirtschaft, Berlin Vom Verlag Die Wirtschaft genehmigte Ausgabe

Alle Rechte vorbehalten

Ohne ausdrückliche Genehmigung des Verlages ist es nicht gestattet, das Buch oder Teile daraus auf fotomechanischem Wege

(Fotokopie, Mikrokopie) zu vervielfältigen.

Gesamtherstellung: Offizin Andersen Nexö, BT V, Leipzig · III/18/38

Umschlaggestaltung: Hanswerner Klein / Reiner Wolfgardt, Opladen 


\section{Inhaltsverzeichnis}

Vorwort $\ldots \ldots \ldots \ldots \ldots \ldots \ldots \ldots \ldots \ldots \ldots \ldots \ldots \ldots \ldots \ldots \ldots \ldots \ldots \ldots, 23$

1. Einführung in die Logik $\ldots \ldots \ldots \ldots \ldots \ldots \ldots \ldots \ldots \ldots \ldots \ldots$

1.1. Aussagen, Variable, Aussageformen .................. 29

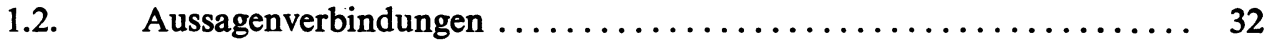

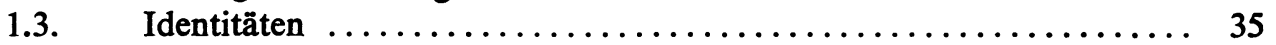

1.3 1. Logisch äquivalente Aussagenverbindungen $\ldots \ldots \ldots \ldots \ldots \ldots \ldots \ldots$

1.3.2. Identitäten in Form einer Implikation $\ldots \ldots \ldots \ldots \ldots \ldots \ldots \ldots \ldots$

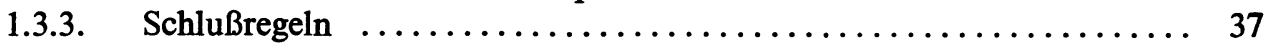

1.4. Elemente der Schaltalgebra $\ldots \ldots \ldots \ldots \ldots \ldots \ldots \ldots \ldots \ldots \ldots \ldots \ldots \ldots$

1.5. Quantifizierung von Aussageformen $\ldots \ldots \ldots \ldots \ldots \ldots \ldots \ldots \ldots \ldots$

Aufgaben ................................ 45

2. Grundbegriffe der Mengenlehre $\ldots \ldots \ldots \ldots \ldots \ldots \ldots \ldots \ldots \ldots$

2.1. Vorbemerkungen $\ldots \ldots \ldots \ldots \ldots \ldots \ldots \ldots \ldots \ldots \ldots \ldots \ldots \ldots \ldots$

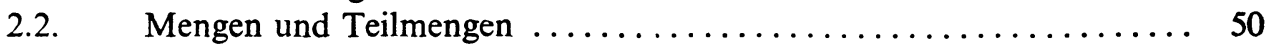

2.3. Mengenoperationen ....................... 53 
2.4. Produktmengen, Relationen $\ldots \ldots \ldots \ldots \ldots \ldots \ldots \ldots \ldots \ldots \ldots \ldots . \ldots \ldots$

2.4.1. Produktmengen, Relationsbegriff $\ldots \ldots \ldots \ldots \ldots \ldots \ldots \ldots \ldots \ldots$

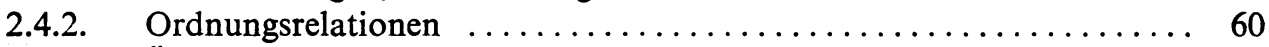

2.4.3. Äquivalenzrelationen, Klasseneinteilungen $\ldots \ldots \ldots \ldots \ldots \ldots \ldots \ldots$

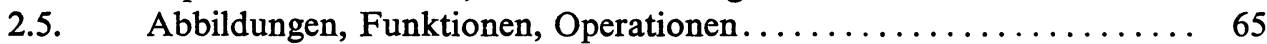

2.6. Gleichmächtigkeit von Mengen, Endlichkeit $\ldots \ldots \ldots \ldots \ldots \ldots \ldots$

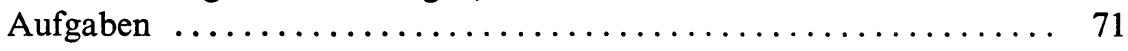

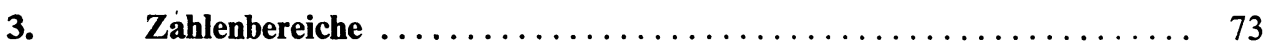

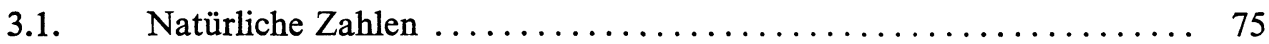

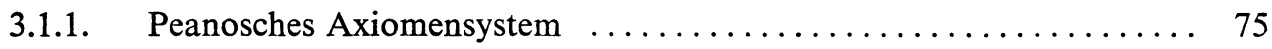

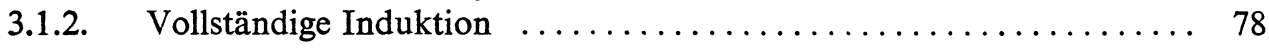

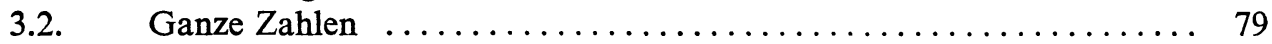

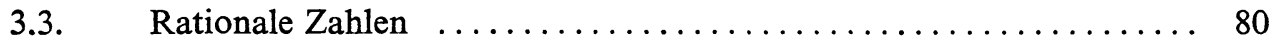

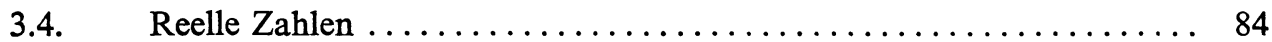

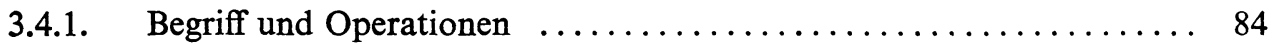

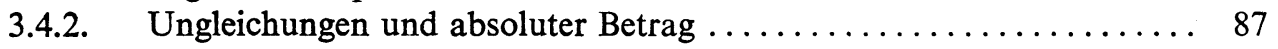

3.4.3. Darstellung reeller Zahlen (Zahlensysteme) $\ldots \ldots \ldots \ldots \ldots \ldots \ldots \ldots 91$

3.5. Komplexe Zahlen............................. 99

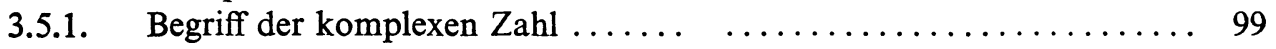

3.5.2. Rechnen mit komplexen Zahlen $\ldots \ldots \ldots \ldots \ldots \ldots \ldots \ldots \ldots \ldots$ 
3.5.3. Polarkoordinaten, trigonometrische Darstellung $\ldots \ldots \ldots \ldots \ldots \ldots \ldots 2$

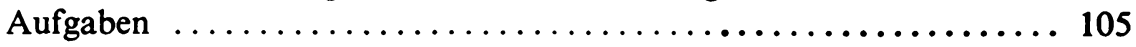

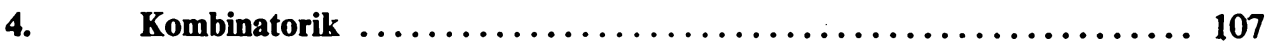

4.1. Summenzeichen $\ldots \ldots \ldots \ldots \ldots \ldots \ldots \ldots \ldots \ldots \ldots \ldots \ldots \ldots$

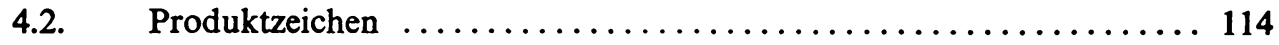

4.3. Aufgaben der Kombinatorik ......................... 116

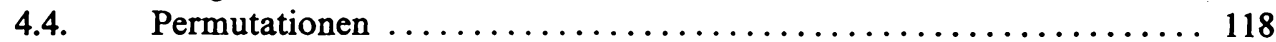

4.4.1. Permutationen ohne Wiederholung $\ldots \ldots \ldots \ldots \ldots \ldots \ldots \ldots \ldots \ldots \ldots \ldots \ldots$

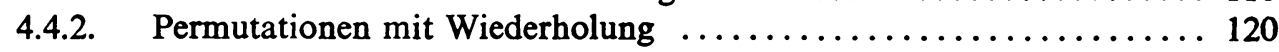

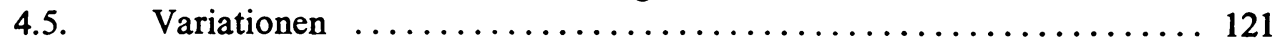

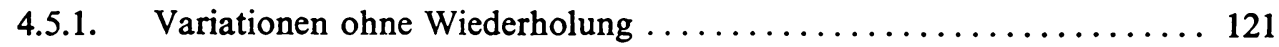

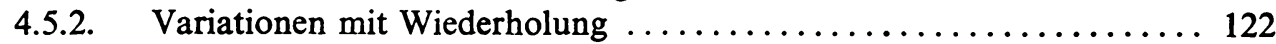

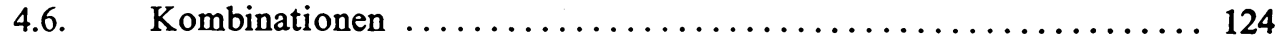

4.6.1. Kombinationen ohne Wiederholung $\ldots \ldots \ldots \ldots \ldots \ldots \ldots \ldots \ldots . \ldots \ldots$

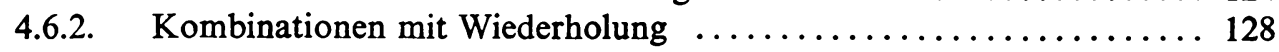

4.7. Binomial- und Polynomialsatz $\ldots \ldots \ldots \ldots \ldots \ldots \ldots \ldots \ldots \ldots \ldots \ldots \ldots \ldots \ldots$

4.7.1. Eigenschaften des Eulerschen Symbols $\left(\begin{array}{l}n \\ k\end{array}\right) \ldots \ldots \ldots \ldots \ldots \ldots \ldots 131$

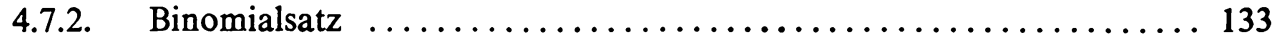

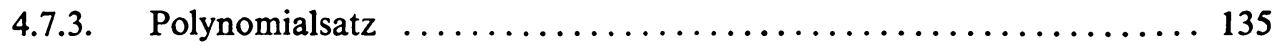

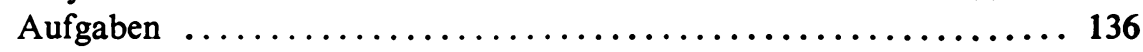


5. Lineare Algebra $\ldots \ldots \ldots \ldots \ldots \ldots \ldots \ldots \ldots \ldots \ldots \ldots \ldots \ldots, 141$

5.1. Matrixbegriff und spezielle Matrizen $\ldots \ldots \ldots \ldots \ldots \ldots \ldots \ldots \ldots$

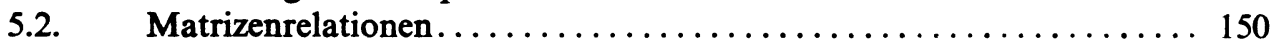

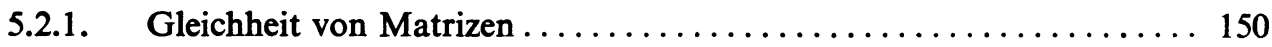

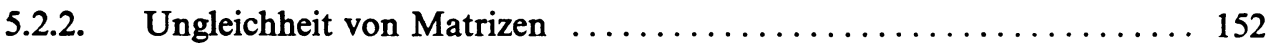

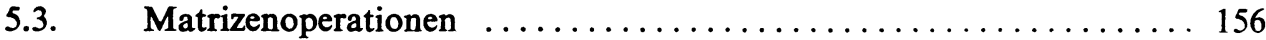

5.3.1. Transponieren.................................... 156

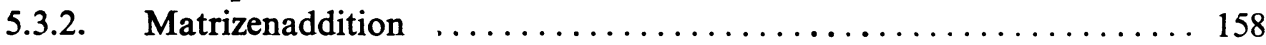

5.3.3. Matrizensubtraktion ............................. 162

5.3.4. Multiplikation einer Matrix mit einem Skalar .............. 164

5.3.5. Multiplikation eines Zeilenvektors mit einem Spaltenvektor (Skalar-

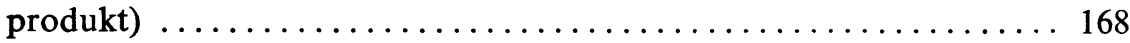

5.3.6. Multiplikation von Matrizen $\ldots \ldots \ldots \ldots \ldots \ldots \ldots \ldots \ldots \ldots \ldots \ldots$

5.4. Linearkombination von Vektoren .................... 178

5.5. Lineare Abhängigkeit und lineare Unabhängigkeit von Vektoren . . . 182

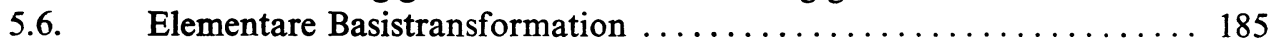

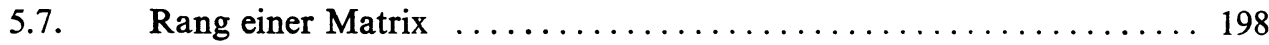

5.8. Konvexe Mengen ............................... 200

5.9. Lineare Gleichungssysteme....................... 205

5.9.1. Begriff des linearen Gleichungssystems $\ldots \ldots \ldots \ldots \ldots \ldots \ldots \ldots 20 \ldots$

5.9.2. Lösbarkeit linearer Gleichungssysteme................... 206

5.9.3. Lineare Gleichungssysteme mit genau einer Lösung ........... 209

5.9.4. Lineare Gleichungssysteme mit unendlich vielen Lösungen ........ 211

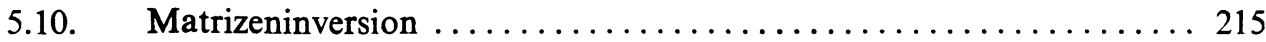




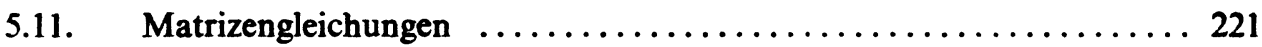

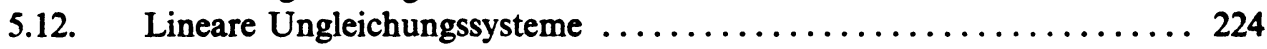

5.12.1. Begriff des linearen Ungleichungssystems $\ldots \ldots \ldots \ldots \ldots \ldots \ldots \ldots 224$

5.12.2. Normales Ungleichungssystem mit beschränkter Lösungsmenge . . . . 227

5.13. Determinanten ................................ 233

5.14. Quadratische Formen und Definitheit $\ldots \ldots \ldots \ldots \ldots \ldots \ldots \ldots . \ldots \ldots 1$

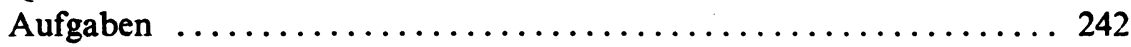

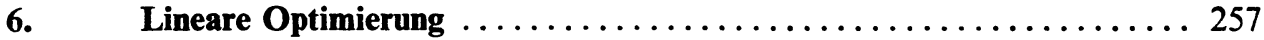

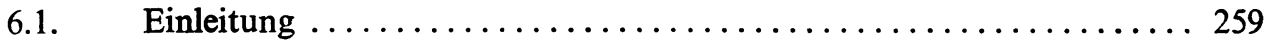

6.2. Lineare Optimierungsmodelle und die Normalform der linearen Opti-

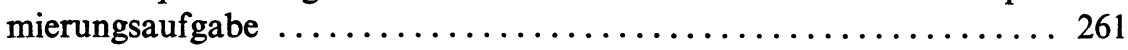

6.2.1. Lineare Optimierungsmodelle ...................... 261

6.2.2. Normalform der linearen Optimierungsaufgabe $\ldots \ldots \ldots \ldots \ldots \ldots 262$

6.3. Graphische Lösung von linearen Optimierungsaufgaben in zwei Variablen ................................... 269

6.4. Grundlegende Eigenschaften linearer Optimierungsaufgaben $\ldots . \ldots 276$

6.5. Simplexmethode .................................. 278

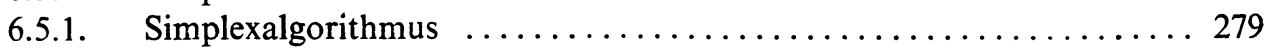

6.5.2. Zur numerischen Durchführung des Simplexalgorithmus ........ 284

6.5.3. Erzeugung einer ersten zulässigen Basislösung und der zugehörigen kanonischen Form - die Phase I der Simplexmethode .......... 295 
6.5.4. Numerische Durchführung der zwei Phasen der Simplexmethode.... 301

6.6. Dualitätstheorie der linearen Optimierung . . . . . . . . . . . . . 308

6.6.1. Paare dualer linearer Optimierungsaufgaben................ 309

6.6.2. Eigenschaften von Paaren dualer linearer Optimierungsaufgaben . . . 312

6.6.3. Ökonomische Interpretation eines Paares dualer linearer Optimierungsaufgaben ..................................... 317

6.7. Dualer Simplexalgorithmus ........................ 318

6.7.1. Theoretische Betrachtungen zum dualen Simplexalgorithmus ....... 319

6.7.2. Zur numerischen Durchführung des dualen Simplexalgorithmus .... 322

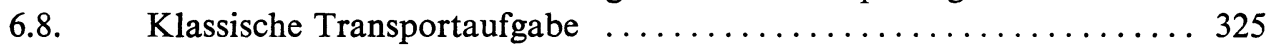

6.8.1. Problemstellung und Modellkonstruktion $\ldots \ldots \ldots \ldots \ldots \ldots \ldots 325$

6.8.2. Eigenschaften der klassischen Transportaufgabe und die Erzeugung zulässiger Basislösungen ............................. 329

6.8.3. Erzeugung einer optimalen zulässigen Basislösung . .......... 338

6.8.4. Zur numerischen Durchführung des Lösungsverfahrens für die klas-

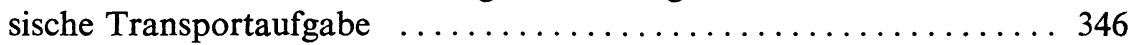

6.9. Parametrische lineare Optimierung .................. 350

6.9.1. Lineare Abhängigkeit der Bewertungskoeffizienten von einem Parameter ................................. 351

6.9.2. Lineare Abhängigkeit des Erfordernisvektors von einem Parameter . . 359

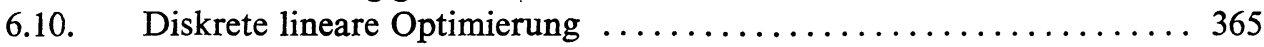

6.10.1. Schnittebenenverfahren von Gomory $\ldots \ldots \ldots \ldots \ldots \ldots \ldots \ldots \ldots \ldots$

Aufgaben .................................... 370 


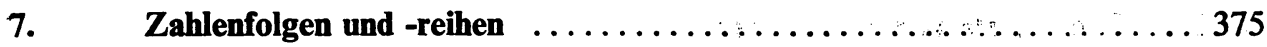

7.1. Begriff der Zahlenfolge, spezielle Zahlenfolgen $\ldots \ldots \ldots \ldots \ldots \ldots \ldots 377$

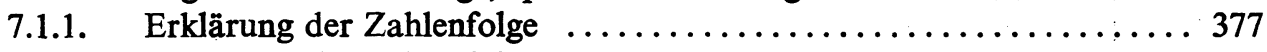

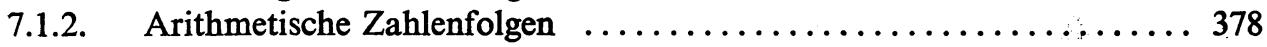

7.1.3. Differenzenfolgen $\ldots \ldots \ldots \ldots \ldots \ldots \ldots \ldots \ldots \ldots \ldots \ldots \ldots \ldots \ldots \ldots . \ldots \ldots$

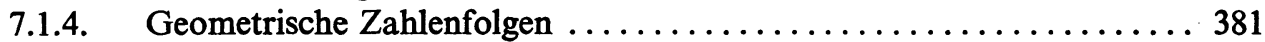

7.1.5. Beschränkte und monotone Zahlenfolgen ............... 383

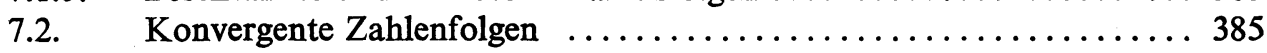

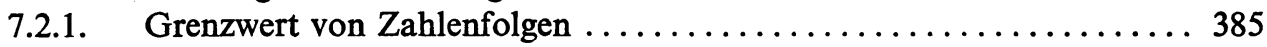

7.2.2. Eigenschaften konvergenter Zahlenfolgen $\ldots \ldots \ldots \ldots \ldots \ldots \ldots \ldots \ldots . \ldots \ldots$

7.2.3. Divergente Zahlenfolgen $\ldots \ldots \ldots \ldots \ldots \ldots \ldots \ldots \ldots \ldots \ldots \ldots \ldots \ldots \ldots$

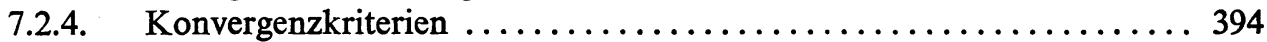

7.3. Zahlenreihen .............................. 397

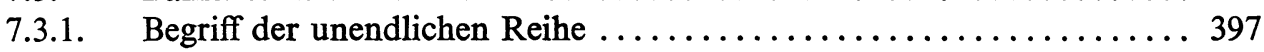

7.3.2. Summe einer unendlichen Reihe $\ldots \ldots \ldots \ldots \ldots \ldots \ldots \ldots \ldots \ldots \ldots$

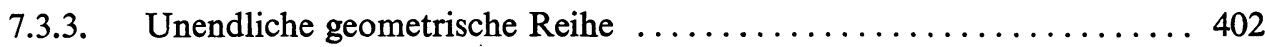

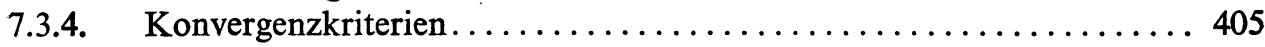

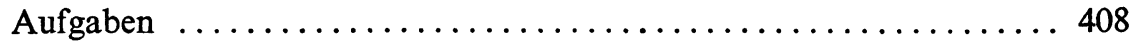

8. Differentialrechnung für Funktionen mit einer unabhängigen Variablen 413

8.1. Funktionen mit einer unabhängigen Variablen $\ldots \ldots \ldots \ldots \ldots \ldots \ldots 15$

8.1.1. Eigenschaften und Typen von Funktionen $\ldots \ldots \ldots \ldots \ldots \ldots \ldots \ldots$ 


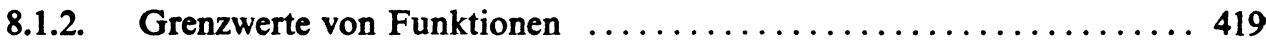

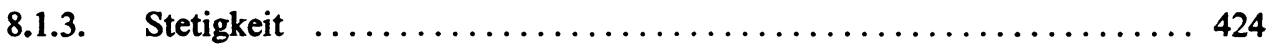

8.2. Differenzierbarkeit ............................ 430

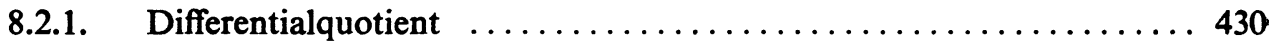

8.2.2. Differentiationsregeln $\ldots \ldots \ldots \ldots \ldots \ldots \ldots \ldots \ldots \ldots \ldots \ldots, 433$

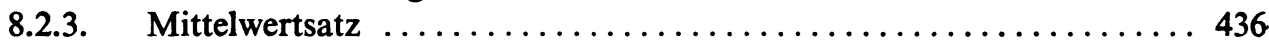

8.2.4. Differentiale ................................ 437

8.2.5. Ableitungen höherer Ordnung ....................... 440

8.3. Satz von Taylor; Taylorsche Reihen $\ldots \ldots \ldots \ldots \ldots \ldots \ldots \ldots \ldots . \ldots 41$

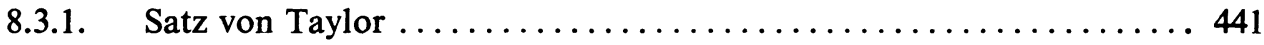

8.3.2. Taylorsche Reihen ............................ 448

8.3.3. Exponential-, Logarithmus- und Potenzfunktion $\ldots \ldots \ldots \ldots \ldots . \ldots 45$

8.4. Anwendungen der Differentialrechnung zur Untersuchung von Funk-

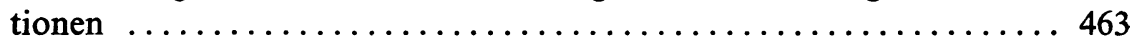

8.4.1. Relative und absolute Extrema ...................... 463

8.4.2. Monotonie, Konvexität, Konkavität .................... 469

8.5. Ökonomische Anwendungen der Differentialrechnung .......... 473

8.5.1. Optimale Losgröße .............................. 473

8.5.2. Optimale Nutzungsdauer .......................... 476

8.5.3. Optimale Laufzeit von Fördersonden $\ldots \ldots \ldots \ldots \ldots \ldots \ldots \ldots . \ldots 48$

8.5.4. Optimale Ankunftsintensität von Schiffen $\ldots \ldots \ldots \ldots \ldots \ldots \ldots 481$

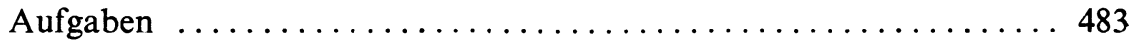


9. Differentialrechnung für Funktionen mit mehreren unabhängigen

Variablen

9.1. Funktionen mit mehreren unabhängigen Variablen . . . . . . .... 489

9.1.1. Grundbegriffe und geometrische Darstellung .............. 489

9.1.2. Grenzwerte und Stetigkeit $\ldots \ldots \ldots \ldots \ldots \ldots \ldots \ldots \ldots \ldots \ldots \ldots \ldots \ldots$

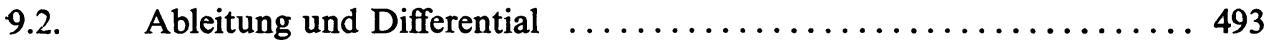

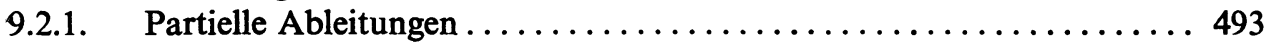

9.2.2. Vollständiges Differential ...................... 495

9.2.3. Partielle Ableitungen höherer Ordnung $\ldots \ldots \ldots \ldots \ldots \ldots \ldots \ldots \ldots . \ldots 4$

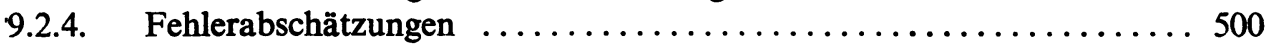

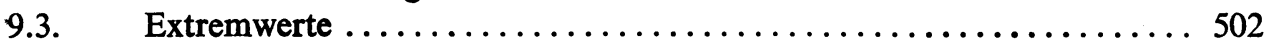

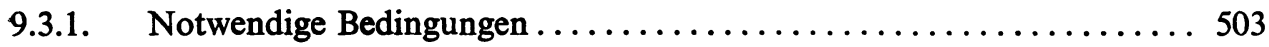

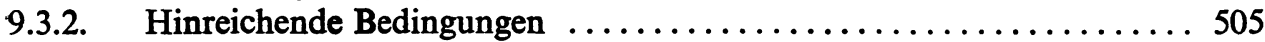

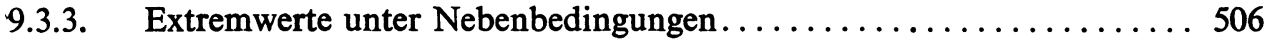

9.3.4. Methode der kleinsten Quadratsumme................ 510

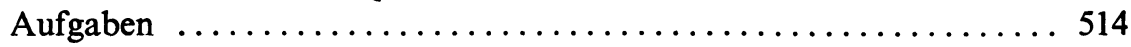

10. Integralrechnung mit einer unabhängigen Variablen $\ldots \ldots \ldots \ldots \ldots 517$

10.1. Unbestimmtes Integral $\ldots \ldots \ldots \ldots \ldots \ldots \ldots \ldots \ldots \ldots \ldots \ldots \ldots \ldots$

10.1.1. Stammfunktion. ............................ 519

10.1.2. Grundregeln zur Ermittlung unbestimmter Integrale . . . . . . . 520 
10.1.3. Partialbruchzerlegung rationaler Funktionen $\ldots \ldots \ldots \ldots \ldots \ldots \ldots 525$

10.1.4. Integration rationaler Funktionen ..................... 530

10.1.5. Integration einiger spezieller Funktionen $\ldots \ldots \ldots \ldots \ldots \ldots \ldots \ldots 533$

10.2. Bestimmtes Integral . . . . . . . . . . . . . . . . . . . . . . 534

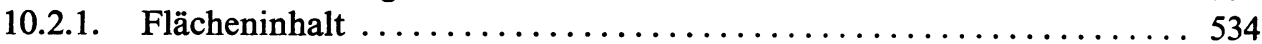

10.2.2. Bestimmtes (Riemannsches) Integral $\ldots \ldots \ldots \ldots \ldots \ldots \ldots \ldots . \ldots 536$

10.2.3. Integrierbarkeit monotoner und stetiger Funktionen $\ldots \ldots \ldots \ldots \ldots 540$

10.2.4. Mittelwertsätze der Integralrechnung $\ldots \ldots \ldots \ldots \ldots \ldots \ldots \ldots . \ldots 51$

10.2.5. Hauptsatz der Differential- und Integralrechnung ............. 542

10.2.6. Rechnen mit bestimmten Integralen ...................... 544

10.3. Anwendungen der Integralrechnung $\ldots \ldots \ldots \ldots \ldots \ldots \ldots \ldots, 546$

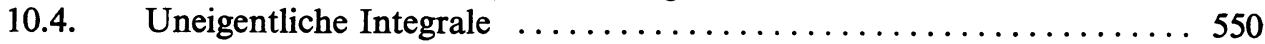

10.4.1. Integrale über unbeschränkte Intervalle ................. 550

10.4.2. Integrale von nicht beschränkten Funktionen ............... 552

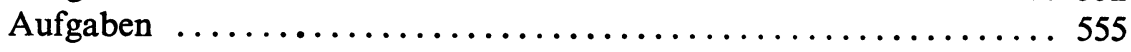

11. Lineare Differential- und Differenzengleichungen $\ldots \ldots \ldots \ldots \ldots 559$

11.1. Lineare Differentialgleichungen $\ldots \ldots \ldots \ldots \ldots \ldots \ldots \ldots \ldots \ldots, 561$

11.1.1. Allgemeine Bemerkungen und Definitionen ................. 561

11.1.2. Differentialgleichung 1. Ordnung $\ldots \ldots \ldots \ldots \ldots \ldots \ldots \ldots \ldots 62$

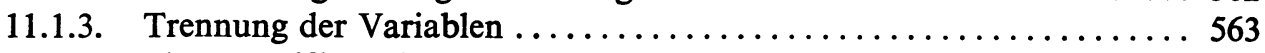

11.1.4. Lineare Differentialgleichung 1 . Ordnung $\ldots \ldots \ldots \ldots \ldots \ldots \ldots . \ldots 66$

11.1.5. Sätze über die Lösungen der homogenen linearen Differentialgleichungen n-ter Ordnung mit konstanten Koeffizienten ............ 570 
11.1.6. Homogene lineare Differentialgleichung 2. Ordnung $\ldots \ldots \ldots \ldots \ldots 574$

11.1.7. Homogene lineare Differentialgleichung n-ter Ordnung . . . . . . . 578

11.1.8. Allgemeine Lösung der inhomogenen linearen Differentialgleichung . . 580

11.1.9. Methode der Variation der Konstanten für die inhomogene lineare Differentialgleichung 2 . Ordnung $\ldots \ldots \ldots \ldots \ldots \ldots \ldots \ldots \ldots \ldots \ldots$

11.1.10. Variation der Konstanten für die inhomogene lineare Differentialglei-

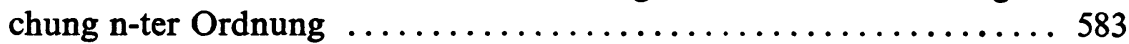

11.1.11. Spezielle Lösungsansätze zur Bestimmung einer partikulären Lösung der inhomogenen linearen Differentialgleichung ........... 585

11.1.12. Systeme von linearen Differentialgleichungen 1. Ordnung . . . ..... 589

11.1.13. Homogene Systeme von linearen Differentialgleichungen 1. Ordnung mit konstanten Koeffizienten ........................ 590

11.1.14. Inhomogene Systeme von linearen Differentialgleichungen 1. Ordnung mit konstanten Koeffizienten ..................... 596

11.1.15. Eliminationsverfahren zur Lösung von linearen Differentialgleichungs-

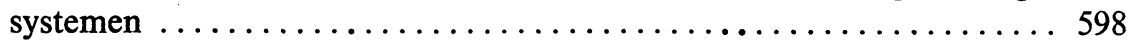

11.1.16. Ökonomische Anwendungen von Differentialgleichungen . . . . . . 600

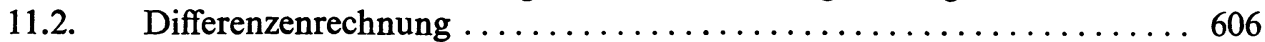

11.2.1. Funktion und ihre Differenzen . . . . . . . . . . . . . . . . . 606

11.2.2. Eigenschaften des Differenzenoperators . . . . . . . . . . . . 610

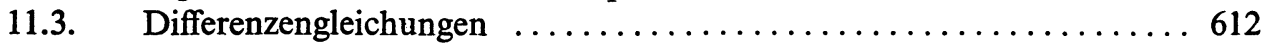

11.3.1. Definitionen ............................. 612

11.3.2. Existenz- und Eindeutigkeitssatz für lineare Differenzengleichungen .. 615

11.3.3. Allgemeine Sätze über lineare Differenzengleichungen mit konstanten

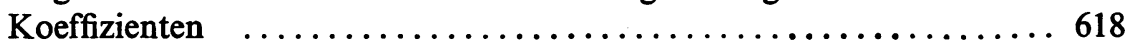


11.3.4. Lineare Differenzengleichung 1. Ordnung mit konstanten Koeffizien-

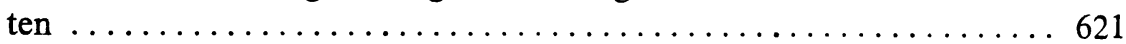

11.3.5. Homogene lineare Differenzengleichung 2. Ordnung mit konstanten Koeffizienten...........................6 623

11.3.6. Homogene lineare Differenzengleichung n-ter Ordnung mit konstanten Koeffizienten............................. 627

11.3.7. Inhomogene lineare Differenzengleichung n-ter Ordnung mit konstanten Koeffizienten .......................... 628

11.3.8. Systeme linearer Differenzengleichungen 1. Ordnung mit konstanten

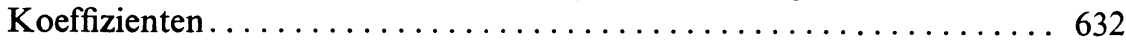

11.4. Zusammenhang zwischen Differenzen- und Differentialgleichungen ... 634

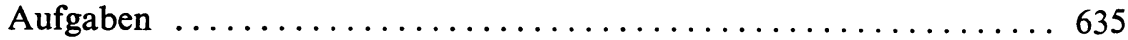

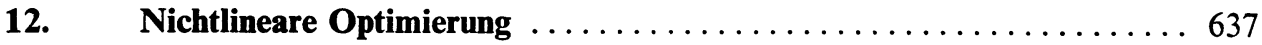

2.1 . Problemstellungen der nichtlinearen Optimierung . . . . . . . . . . 640

12.1.1. Allgemeine Aufgabe der nichtlinearen Optimierung . . . . . . . . . 640

12.1.2. Graphische Lösung von nichtlinearen Optimierungsaufgaben in zwei Variablen ..................................6 642

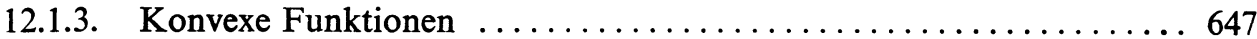

12.1.4. Globale und relative Extrema $\ldots \ldots \ldots \ldots \ldots \ldots \ldots \ldots \ldots \ldots \ldots \ldots$

12.1.5. Besonderheiten der nichtlinearen Optimierung $\ldots \ldots \ldots \ldots \ldots \ldots \ldots 4$

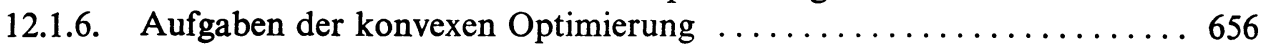

12.1.7. Hyperbolische Optimierung $\ldots \ldots \ldots \ldots \ldots \ldots \ldots \ldots \ldots \ldots \ldots \ldots$ 
12.1.8. Quadratische Optimierung $\ldots \ldots \ldots \ldots \ldots \ldots \ldots \ldots \ldots \ldots \ldots \ldots \ldots 6 . \ldots \ldots$

12.2. Approximationsmethoden für Probleme mit trennbaren Funktionen .. 664

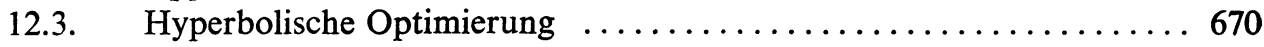

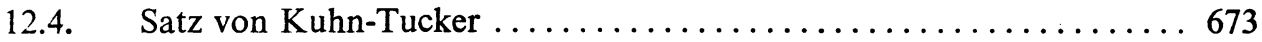

12.4.1. Satz von Kuhn-Tucker für konvexe Probleme .............. 673

12.4.2. Bedingungen von Kuhn-Tucker für quadratische Probleme ........ 676

12.5. Quadratische Optimierung $\ldots \ldots \ldots \ldots \ldots \ldots \ldots \ldots \ldots \ldots . \ldots 679$

12.5.1. Allgemeine Aussagen über quadratische Probleme ............. 679

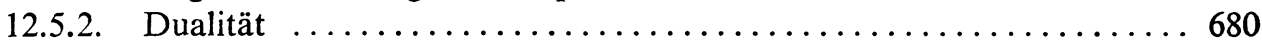

12.6. Gradientenverfahren ............................ 682

12.6.1. Allgemeines Vorgehen der Gradientenverfahren.............. 682

12.6.2. Quadratischer Fall ............................... 684

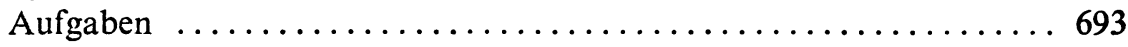

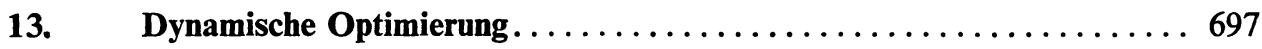

13.1. Stellung der dynamischen Optimierung in der Optimierungstheorie ... 699

13.2. Mehrstufige Entscheidungsprozesse .................... 700

13.2.1. Dynamische Systeme und Mehrstufenprozesse $\ldots \ldots \ldots \ldots \ldots \ldots 700$

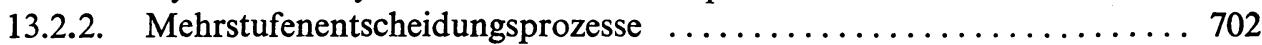

13.2.3. Separable Mehrstufenentscheidungsprozesse $\ldots \ldots \ldots \ldots \ldots \ldots \ldots 703$

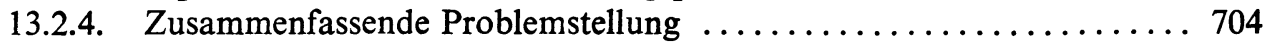

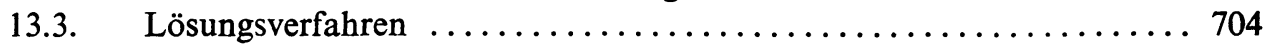

13.3.1. Optimalitätsprinzip . ............................ 704 


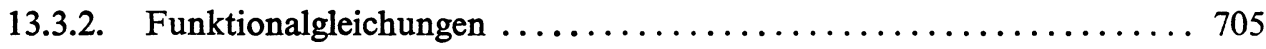

13.3.3. Lösung der Funktionalgleichungen $\ldots \ldots \ldots \ldots \ldots \ldots \ldots \ldots \ldots 70 \ldots \ldots$

13.3.4. Fragen der praktischen Auswertung der Funktionalgleichungen . . . . 708

13.3.5. Umkehrung der Optimierungsrichtung . . . . . . . . . . . . . . 710

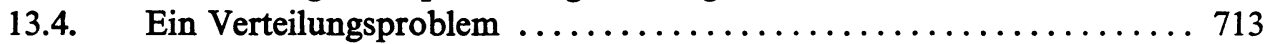

13.5. Wertung des Verfahrens und Ausblick ................... 719

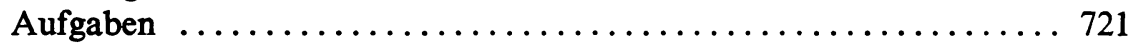

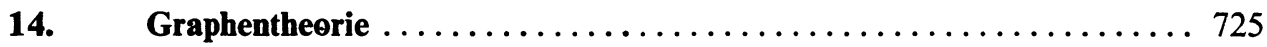

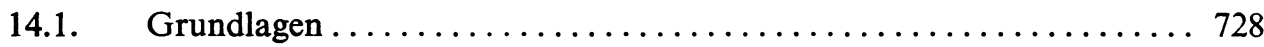

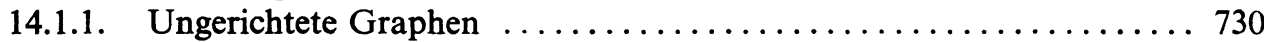

14.1.2. Gerichtete Graphen ............................. 733

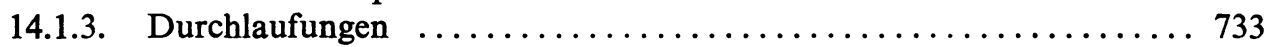

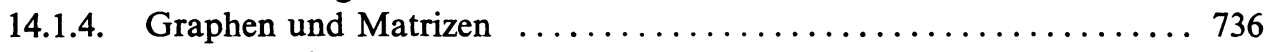

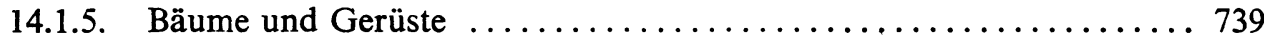

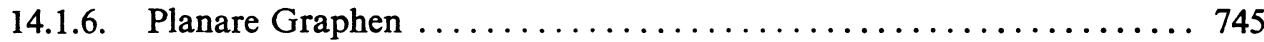

14.2. Anwendungen der Graphentheorie in der Ökonomie ........... 746

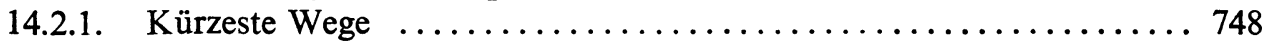

14.2.2. Einiges über Netzplantechnik $\ldots \ldots \ldots \ldots \ldots \ldots \ldots \ldots \ldots \ldots \ldots \ldots \ldots \ldots \ldots$

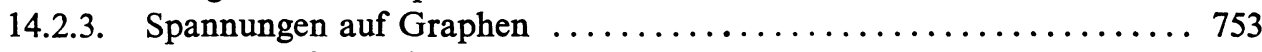

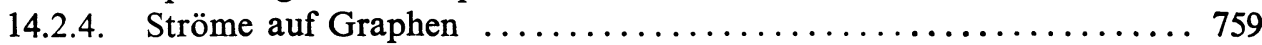

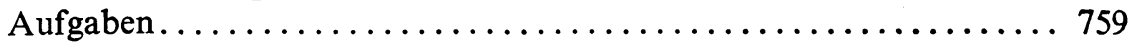


15. Wahrscheinlichkeitsrechnung $\ldots \ldots \ldots \ldots \ldots \ldots \ldots \ldots \ldots \ldots \ldots \ldots \ldots \ldots \ldots \ldots \ldots \ldots \ldots$

15.1. Einführung in die Wahrscheinlichkeitsrechnung $\ldots \ldots \ldots \ldots \ldots \ldots 763$

15.1.1. Gegenstand der Wahrscheinlichkeitsrechnung $\ldots \ldots \ldots \ldots \ldots \ldots \ldots$

15.1.2. Ereignisse und deren Wahrscheinlichkeit ............... 765

15.1.3. Klassische Definition der Wahrscheinlichkeit $\ldots \ldots \ldots \ldots \ldots \ldots \ldots 770$

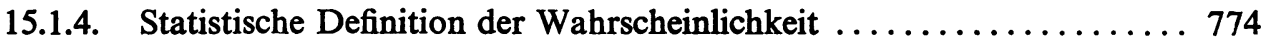

15.1.5. Axiomatische Definition der Wahrscheinlichkeit ............ 776

15.1.6. Rechnen mit Wahrscheinlichkeiten $\ldots \ldots \ldots \ldots \ldots \ldots \ldots \ldots \ldots \ldots 78$

15.1.7. Unabhängigkeit und Abhängigkeit von Ereignissen.......... 786

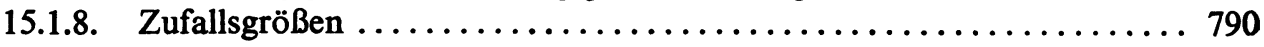

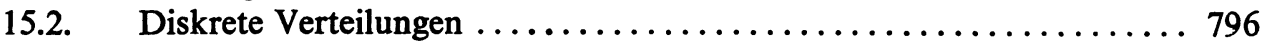

15.2.1. Verteilungsgesetz einer diskreten Zufallsgröße ............. 796

15.2.2. Erwartungswert und Varianz einer diskreten Zufallsgröße ...... 803

15.2.3. Weitere Parameter einer diskreten Zufallsgröße $\ldots \ldots \ldots \ldots \ldots \ldots . \ldots 10$

15.2.4. Erzeugende Funktion einer diskreten Zufallsgröße $\ldots \ldots \ldots \ldots \ldots \ldots 813$

15.2.5. Mehrdimensionale diskrete Zufallsgrößen $\ldots \ldots \ldots \ldots \ldots \ldots \ldots \ldots . \ldots 15$

15.3. Spezielle diskrete Verteilungen ................... 818

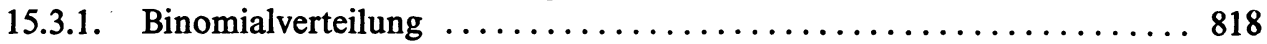

15.3.2. Hypergeometrische Verteilung $\ldots \ldots \ldots \ldots \ldots \ldots \ldots \ldots \ldots \ldots \ldots 25$

15.3.3. Geometrische Verteilung. ....................... 826

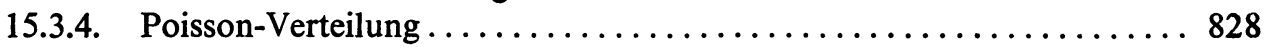

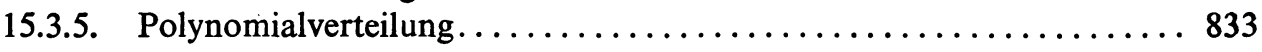

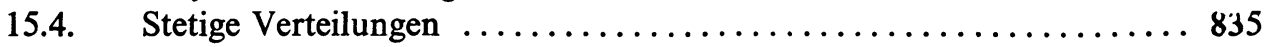

15.4.1. Verteilungsgesetz einer stetigen Zufallsgröße............. 835 


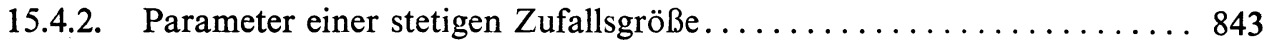

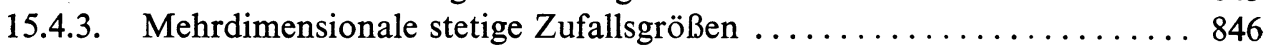

15.5. Spezielle stetige Verteilungen $\ldots \ldots \ldots \ldots \ldots \ldots \ldots \ldots \ldots \ldots \ldots 48$

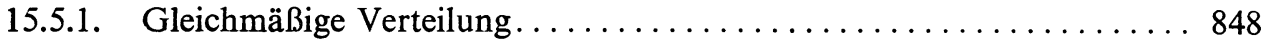

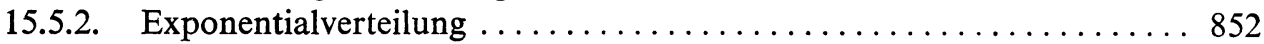

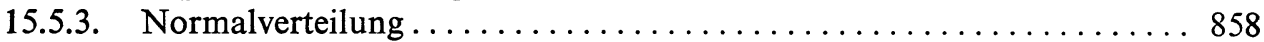

15.5.4. Zweidimensionale Normalverteilung. . . . . . . . . . . . . . . . . 869

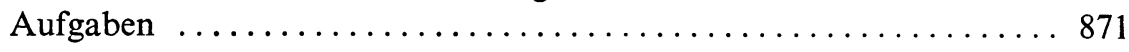

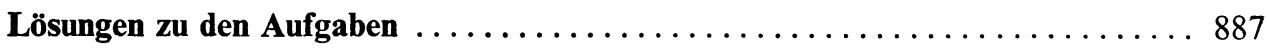

1. Einführung in die Logik $\ldots \ldots \ldots \ldots \ldots \ldots \ldots \ldots \ldots \ldots$

2. Ġundbegriffe der Mengenlehre..................... 890

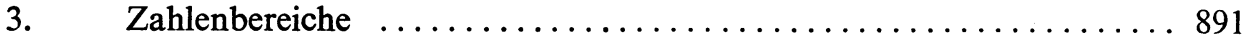

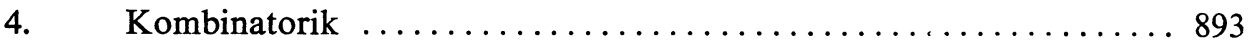

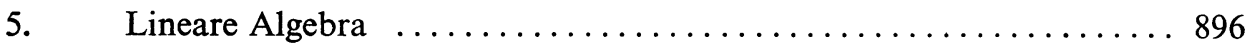

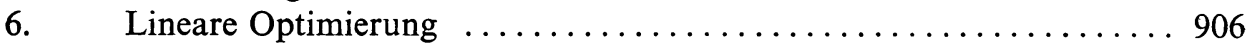

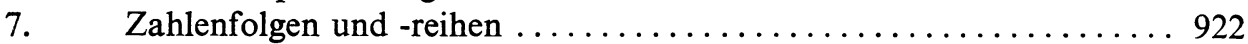

8. Differentialrechnung für Funktionen mit einer unabhängigen Variablen 931

9. Differentialrechnung für Funktionen mit mehreren unabhängigen Variablen ..................................... 934

10. Integralrechnung mit einer unabhängigen Variablen . . . . . . . 936

11. Lineare Differential- und Differenzengleichungen ............ 939

12. Nichtlineare Optimierung ...................... 940 
13. Dynamische Optimierung $\ldots \ldots \ldots \ldots \ldots \ldots \ldots \ldots \ldots \ldots \ldots \ldots, 948$

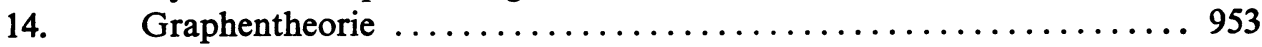

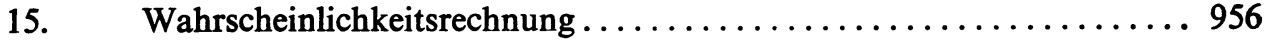

Literaturverzeichnis ................................... 979

Sachwortverzeichnis ................................... 985 


\section{Vorwort}

Wie viele andere Wissenschaftsbereiche werden auch die Wirtschaftswissenschaften in zunehmendem Maße operationeller und bedürfen daher formaler Methoden zur Lösung ihrer Probleme. Entscheidende Voraussetzung für diese Entwicklung ist die Verwendung der mathematischen Theorie: Durch Operationen mit Abstraktionen wie Konstanten, Variablen, Matrizen, Vektoren, Vektorräumen usw. und unter $\mathrm{Zu}$ grundelegung von Axiomen gelingt es, mit Hilfe der Mathematik wirtschaftswissenschaftliche Probleme zu beschreiben und mit Hilfe bestimmter Algorithmen zu lösen. Je komplizierter und vielgestaltiger die quantitative Struktur wirtschaftlicher Phänomene in wirtschaftswissenschaftlicher Forschung und in der Wirtschaftspraxis in Erscheinung treten, um so notwendiger ist die Mathematik zur Beherrschung und Gestaltung des Wirtschaftsablaufs. Die Mathematik mit ihren allgemeinen Formulierungen von Problemen ermöglicht eine kurze präzise Darstellung der Wirklichkeit mit ihren vielseitigen Zusammenhängen, Beziehungen und Abhängigkeiten. Das weite Anwendungsgebiet der Mathematik in den Wirtschaftswissenschaften und einigen Disziplinen, die für die Wirtschaftswissenschaften von Bedeutung sind, soll die folgende Zusammenstellung zeigen:

Entscheidungs- und Spieltheorie

Mengenlehre, mathematische Logik, Kombinatorik, lineare Algebra

Politische Arithmetik (Finanz- und Wirtschaftsarithmetik)

Folgen und Reihen; Potenzrechnen, Logarithmen 
Wachstumstheorie

Lineare Algebra, Differential- und Differenzengleichungen

\section{Programmierung}

Lineare Algebra, lineare und nichtlineare Optimierung, dynamische Optimierung

Input-Output-Analyse

Lineare Algebra (Matrizenrechung, lineare Gleichungen)

Ökonometrie und Statistik

Wahrscheinlichkeitsrechnung, mathematische Statistik, lineare Algebra (Matrizenund Determinantenrechnung, lineare Gleichungssysteme), Methode der kleinsten Quadrate

Netzplantechnik

Graphentheorie

Marktforschung

Mengenlehre, Wahrscheinlichkeitsrechnung, Spieltheorie, lineare Algebra

\section{Operations Research (Unternehmensforschung)}

Lineare und nichtlineare Optimierung, Wahrscheinlichkeitsrechnung, Differentialund Integralrechnung, Kombinatorik, Mengenlehre, lineare Algebra

\section{Kybernetik, elektronische Datenverarbeitung}

Mengenlehre, mathematische Logik, lineare Algebra

Die moderne Wirtschaftstheorie und ihre Anwendung in der volkswirtschaftlichen und betrieblichen Praxis wäre ohne Verwendung der mathematischen Methoden undenkbar; um so mehr erscheint es daher notwendig, den Studenten der Wirtschaftswissenschaften sowie den Studenten der neu begründeten Studienrichtung für Wirtschaftsingenieure und den im Management tätigen Praktikern die für die Theorie notwendigen Grundlagen der Mathematik zu vermitteln.

Das vorliegende Buch bietet dem interessierten Leser den gesamten mathematischen Lehrstoff, der zur Zeit den Studenten an den wirtschaftswissenschaftlichen Hochschulen und Fakultäten und den Wirtschaftsingenieuren geboten wird; zusätzlich dazu wird eine Darstellung der Wahrscheinlichkeitsrechnung gegeben. Bei gewissenhafter Durcharbeitung des in diesem Buch dargebotenen Lehrstoffes und der Übungsaufgaben wird der Leser in der Lage sein, die mathematisch-ökonomische Literatur und die Grundlagen der neuentstandenen wissenschaftlichen Disziplinen wie Kybernetik, Operationsforschung und elektronische Datenverarbeitung zu verstehen und die Mathematik zur Lösung wirtschaftswissenschaftlicher Probleme anzuwenden. Vom Leser selbst werden nur Kenntnisse der elementaren Schulmathematik vorausgesetzt: Das sind vor allem das Rechnen mit allgemeinen Zahlen und das Lösen von 
Gleichungen mit einer oder mehreren Variablen. Zur leichteren Erfassung und Vertiefung des Gelernten hat der Leser die Möglichkeit, sein Wissen an den Übungsaufgaben am Ende jedes Abschnitts zu prüfen. Die entsprechenden Lösungen werden ebenfalls bereitgestellt.

Zunächst werden im ersten Kapitel die Grundlagen der Logik dargestellt. Deren Kenntnis ist nicht nur zum Verständnis der Mathematik notwendig, sondern im Prinzip für jede Wissenschaft wichtig: speziell ist sie besonders interessant für Datenverarbeitung, Kybernetik und Operations Research. In den weiteren Kapiteln 2., 3. und 4. (Mengenlehre, Zahlentheorie und Kombinatorik) werden die Grundlagen dargestellt, die für das Verständnis der nächsten Abschnitte erforderlich sind. Die Grundlagen der mathematischen Modellbildung für Wirtschaftsabläufe finden sich in den Kapiteln bzw. entsprechenden Abschnitten: Lineare Algebra (5.), Zahlenfolgen und -reihen (7.), Differential- und Integralrechnung (8., 9., 10. und 11.) und Graphentheorie (14.). Zur Ermittlung der ökonomischen Optima werden die Methoden der linearen (6.), der nichtlinearen (12.) und der dynamischen Optimierung (13.) sowie die Differentialrechnung (8., 9. und 11.) herangezogen. Wahrscheinlichkeitstheoretische Methoden für die Darstellung der Ungewißheit in den Wirtschaftswissenschaften werden im Kapitel 15. behandelt.

Vorschläge und kritische Anmerkungen bitten wir an den Verlag zu richten.

Westdeutscher Verlag 